\title{
Snake beam: a paraxial arbitrary focal line
}

\author{
Joseph Rosen and Amnon Yariv \\ California Institute of Technology, m/s 128-95, Pasadena, California 91125
}

Received June 14, 1995

\begin{abstract}
The creation of paraxial arbitrary focal lines by a Fourier computer-generated hologram is demonstrated. The desired focal line is represented by a series of connected straight line segments, each of which is implemented by a radial harmonic function located on a different radial portion of the entire hologram. Each subhologram is multiplied by appropriate linear and quadratic phase functions and is shifted by some distance from the center. The two phase factors determine the location of each line segment, while the in-plane shift determines the tilt angle of the segment. (c) 1995 Optical Society of America
\end{abstract}

Recently we introduced a new beam characterized by an almost constant intensity along a finite propagation distance and a beamlike shape in the transverse dimensions. ${ }^{1}$ This beam is generated by a Fourier hologram with the distribution of a radial harmonic function (RHF). We refer to the beam as a sword beam. Unlike with other methods of creating sword beams by Fourier holograms, which are based on annular $^{2}$ or approximately annular ${ }^{3}$ apertures, our Fourier hologram can be realized by a phase-only mask. Unlike with the iterative optimization methods of synthesizing sword beams, ${ }^{4}$ the RHF is an analytical solution with a few characteristic parameters.

In this Letter we make use of the RHF and three of the Fourier hologram's properties in order to create an arbitrary twisting focal line. We do this by composing several holographic elements, each of which creates a small sword-shaped beam somewhere in the space behind the lens (see Fig. 1). Illuminating the complete hologram by a plane wave yields a twisted curve of light, around the front focus, which we term a snake beam.

Assume that a function $g\left(x_{i}, y_{i}\right)$ is placed as a transparency distribution at the rear focal plane (plane $\mathrm{P}_{\mathrm{i}}$ in Fig. 1) of a spherical lens and illuminated by a plane wave. Under the Fresnel approximation, the complex amplitude distribution around the focal plane $\mathrm{P}_{\mathrm{f}}$ is $^{4}$

$$
\begin{aligned}
& u(x, y, z)=\frac{\exp [j k(z+2 f)]}{j \lambda f} \int_{-\infty}^{\infty} \int_{-\infty}^{\infty} g\left(x_{i}, y_{i}\right) \\
& \times \exp \left[\frac{j 2 \pi\left(x x_{i}+y y_{i}\right)}{\lambda f}-\frac{j \pi z\left(x_{i}{ }^{2}+y_{i}{ }^{2}\right)}{\lambda f^{2}}\right] \mathrm{d} x_{i} \mathrm{~d} y_{i} .
\end{aligned}
$$

For convenience, we designate Eq. (1) the focal space transform (FST), such that $u(x, y, z) \equiv \operatorname{FST}\left\{g\left(x_{i}, y_{i}\right)\right\}$.

Three theorems of the FST, proved in Ref. 1, are useful for creating the snake beam. In the following we summarize them in a form suitable for this analysis.

I. Linear phase theorem: If $\operatorname{FST}\left\{g\left(x_{i}, y_{i}\right)\right\}=$ $u(x, y, z)$, then

$$
\begin{aligned}
\operatorname{FST}\left\{g\left(x_{i}, y_{i}\right) \exp \left[j 2 \pi\left(\xi x_{i}+\eta y_{i}\right)\right\}\right. & \\
= & u(x-\lambda f \xi, y-\lambda f \eta, z) .
\end{aligned}
$$

That is, multiplying $g\left(x_{i}, y_{i}\right)$ by a linear phase function shifts the longitudinal beam laterally by distances $(\lambda f \xi, \lambda f \eta)$ directly related to the phase constants.

II. Quadratic phase theorem: If $\operatorname{FST}\left\{g\left(x_{i}, y_{i}\right)\right\}=$ $u(x, y, z)$, then

$$
\begin{aligned}
\left|\operatorname{FST}\left\{g\left(x_{i}, y_{i}\right) \exp \left[j 2 \pi \gamma\left(x_{i}{ }^{2}+y_{i}{ }^{2}\right)\right]\right\}\right|^{2} & \\
& =\left|u\left(x, y, z-2 \lambda f^{2} \gamma\right)\right|^{2} .
\end{aligned}
$$

That is, multiplying $g\left(x_{i}, y_{i}\right)$ by a quadratic phase function shifts the longitudinal beam along the $z$ axis by a distance $2 \lambda f^{2} \gamma$ directly related to the phase constant.

III. Lateral shift theorem: If $\operatorname{FST}\left\{g\left(x_{i}, y_{i}\right)\right\}=$ $u(x, y, z)$, then

$$
\begin{gathered}
\left|\operatorname{FST}\left\{g\left(x_{i}-\alpha, y_{i}-\beta\right)\right\}\right|^{2}=\left|u\left(x-\frac{z \alpha}{f}, y-\frac{z \beta}{f}, z\right)\right|^{2} \\
=|u(\bar{x} \sec \theta, \bar{y} \sec \varphi, \bar{z} \cos \theta \cos \varphi)|^{2}
\end{gathered}
$$

where $\tan \theta=\alpha / f, \tan \varphi=\beta / f$, and $(\bar{x}, \bar{y}, \bar{z})$ is the tilted coordinates system, at an angle $\theta$ from the $x z$ plane and $\varphi$ from the $y z$ plane. This theorem states that when the input function is shifted laterally the output beam is rotated around the focal point by angles $(\theta, \varphi)$, whose tangents are directly related to the lateral shift $(\alpha, \beta)$. The longitudinal dimension of

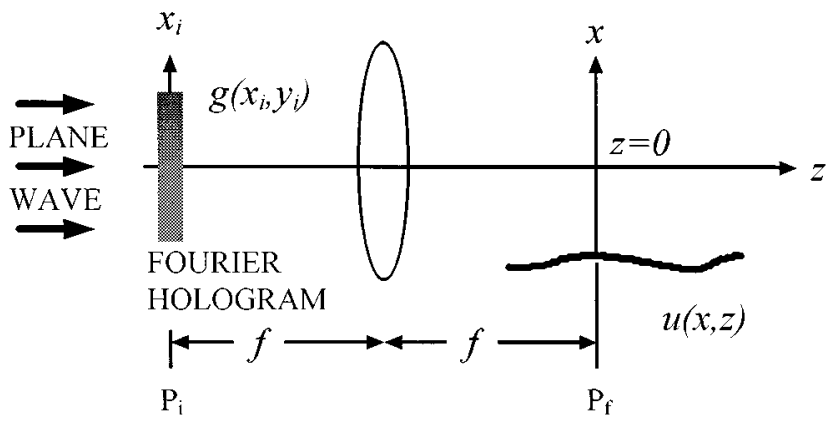

Fig. 1. Schematic of the system used to obtain arbitrary paraxial focal lines. An example of a snake beam is shown by the thick line. 
the rotated beam is stretched by the factor $\sec \theta \sec \varphi$, while the lateral dimensions $(\bar{x}, \bar{y})$ are shrunk by the factors $\cos \theta$ and $\cos \varphi$, respectively.

From these three theorems it follows that if $u(z)$ is a sword beam, it can be shifted laterally, shifted along the $z$ axis, and tilted by some angle, respectively. In other words, as long as we stay in the paraxial regime (the basic assumption of the Fresnel approximation) we can design the sword beam in any place and tilt angle in the three-dimensional space. In practice the beam's location is limited to the space defined by a hologram's maximal diffraction angle of $\pm 18^{\circ}$ from the optical axis (the paraxial regime).

Using the theory of stationary phase approximation, we found that, among other functions, the following phase-only RHF (Ref. 1)

$$
\begin{aligned}
g\left(r_{i}\right) & =\exp \left[j 2 \pi\left(r_{i} / b\right)^{4}\right], \quad R_{1} \leq r_{i} \leq R_{2}, \\
r_{i} & =\sqrt{x_{i}{ }^{2}+y_{i}{ }^{2}}
\end{aligned}
$$

creates a sword beam if $g\left(r_{i}\right)$ is located at plane $\mathrm{P}_{\mathrm{i}}$. Note that $g\left(r_{i}\right)$ is identical to a spherical aberration factor, suitably isolated in a ring. The longitudinal intensity distribution, around the front focal point, resulting from this hologram is

$$
|u(z)|^{2}=\left|\operatorname{FST}\left\{g\left(r_{i}\right)\right\}\right|_{x=y=0}^{2} \approx \begin{cases}C & z_{1} \leq z \leq z_{2} \\ \ll C & \text { otherwise }\end{cases}
$$

where $C$ is some constant. We have also shown that $z_{2} \cong 4 \lambda f^{2} R_{2}{ }^{2} / b^{4}$, and, in the case $R_{1}=0, z_{1} \cong 2 \lambda f^{2} / b^{2}$; otherwise $z_{1} \cong 4 \lambda f^{2} R_{1}^{2} / b^{4}$. It follows from these relations that

$$
\left|z_{2}-z_{1}\right| \equiv \Delta z \cong \frac{8 \lambda f^{2} R_{0} \Delta R}{b^{4}}
$$

where $\Delta R=\left|R_{2}-R_{1}\right|$ and $R_{0}=\left(R_{2}+R_{1}\right) / 2$. Relation (7) is the relation between the sword beam's length and the radial interval of the hologram.

The snake beam will be composed of a collection of $N$ connected sword beams, each at a specified location, length, and tilt. Suppose, for example, that the desired beam curve is that shown on the right-hand side of Fig. 1 as the thick line. The first step of the design process is to approximate the curve by a collection of straight lines, as shown in Fig. 2(a). A tradeoff exists between the need to approximate the original curve with as large a number as possible of straight lines and the limited resolution and aperture diameter of the single holographic mask. As mentioned above, each line is implemented by a different radial portion of the mask. From relation (7) we realize that the length of each sword beam is proportional to the width of the radial portion $\Delta R$ and its average radius $R_{0}$. Therefore the most efficient order is to implement the shortest line by the most internal radial portion of the hologram, to continue in increasing order, and to finish by implementing the longer line by the most external radial portion.
Each sword beam is characterized by four parameters: $L$, its length projected onto the $z$ axis; $h=$ $\left(h_{x}, h_{y}\right)$, its lateral shift from the $z$ axis; $d$, its longitudinal shift (measured from the focal point to the beginning of the sword); and $\theta=\left(\theta_{x}, \theta_{y}\right)$, the tilt angle between the beam and the $z$ axis. For each straight line in Fig. 2(a) we recorded all four parameters. The overall hologram is given by a collection of $N$ RHF's, each of which occupies one radial distance, laterally shifted [by $\left(\alpha_{n}, \beta_{n}\right)$ ], multiplied by a linear phase [with the parameters $\left.\left(\xi_{n}, \eta_{n}\right)\right]$, and multiplied by a quadratic phase (with the parameter $\gamma_{n}$ ). The transparency distribution of the complete hologram is given by

$$
\begin{aligned}
g\left(x_{i}, y_{i}\right)= & \sum_{n=1}^{N} \operatorname{rect}\left\{\frac{\left[\left(x_{i}-\alpha_{n}\right)^{2}+\left(y_{i}-\beta_{n}\right)^{2}\right]^{1 / 2}-R_{n}}{\Delta R_{n}}\right\} \\
& \times \exp \left(j 2 \pi \left\{\frac{\left[\left(x_{i}-\alpha_{n}\right)^{2}+\left(y_{i}-\beta_{n}\right)^{2}\right]^{2}}{b^{4}}\right.\right. \\
& \left.\left.+\xi_{n} x_{i}+\eta_{n} y_{i}+\gamma_{n} r_{i}{ }^{2}\right\}\right)
\end{aligned}
$$

(a)

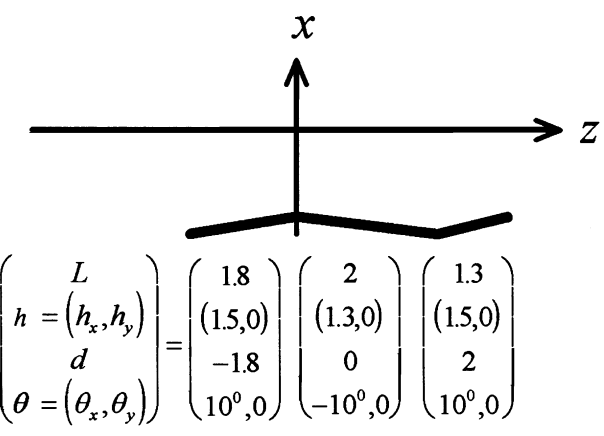

(b)

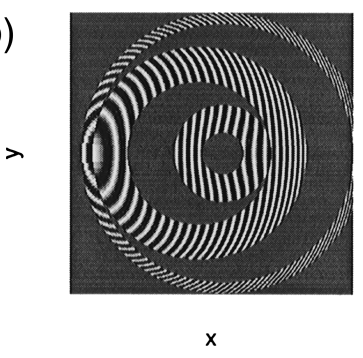

(c)

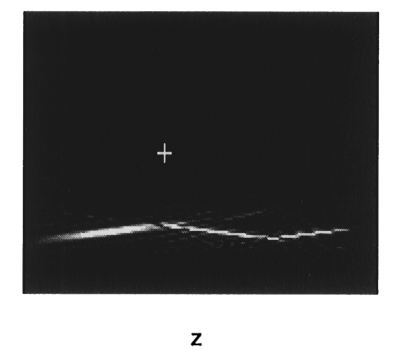

Fig. 2. (a) Approximation of straight lines of the focal curve shown in Fig. 1. All the distances are in arbitrary units. (b) The real part of the Fourier hologram (128 $\times$ 128 pixels) that generates the beam shown in (a). (c) Output intensity distribution in the $x-z$ plane obtained by computer simulation of the system shown in Fig. 1 . The small white cross indicates the front focus location. 
(a)

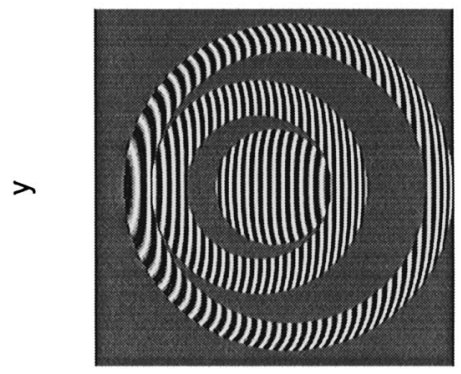

$\mathbf{x}$

(b)

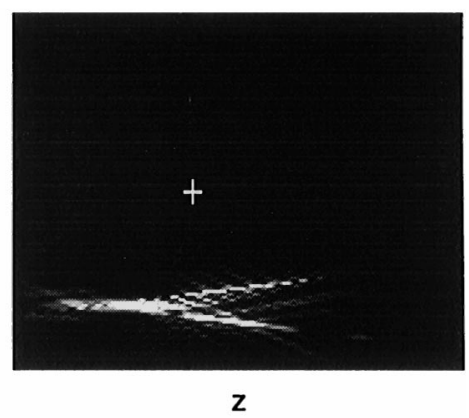

Fig. 3. (a) Real part of the Fourier hologram $(128 \times$ 128 pixels) that generates the treelike beam. (c) Output intensity distribution of the treelike beam in the $x-z$ plane obtained by computer simulation.

where

$$
\begin{gathered}
\operatorname{rect}\left(\frac{x}{2 \Delta}\right) \equiv \begin{cases}1 & -\Delta \leq x \leq \Delta \\
0 & \text { otherwise }\end{cases} \\
R_{n}=\frac{b^{2}}{2 f \sqrt{\lambda}}\left(z_{0}+\frac{L_{n}}{2}+\sum_{i=1}^{n-1} L_{i}\right)^{1 / 2}+\sum_{i=1}^{n} \max \left\{\left|\alpha_{n}\right|,\left|\beta_{n}\right|\right\} \\
\Delta R_{n}=\frac{L_{n} b^{4}}{8 \lambda f^{2} R_{n}}, \quad z_{0}=\frac{2 \lambda f^{2}}{b^{2}} \\
\gamma_{n}=\frac{d_{n}-\left(z_{0}+\sum_{i=1}^{n} L_{i}\right)}{2 \lambda f^{2}}, \quad \xi_{n}=\frac{h_{x, n}}{\lambda f} \\
\eta_{n}=\frac{h_{y, n},}{\lambda f}, \quad \alpha_{n}=f \tan \theta_{x, n}
\end{gathered}
$$

and $\beta_{n}=f \tan \theta_{y, n}$.

All the above parameters are obtained by interpreting the three theorems and relation (7). It is desirable that all parts of the hologram have the same value of the parameter $b$, in order to guarantee the same intensity level along the curve. The precise value of $b$ may be determined such that on one hand it will not be too small in the sense that any local frequency somewhere over the hologram will not exceed the sampling rate of the computer's digital grid and on the other hand it will not be so large that at some radial portion the RHF will not have enough cycles to justify the use of the stationary phase approximation.

Using the parameters of Fig. 2(a), we synthesized the hologram following the procedure of Eq. (8). The real part of the resulting Fourier hologram is shown in Fig. 2(b). Illumination of this mask, located at plane $\mathrm{P}_{\mathrm{i}}$, by a plane wave yields the desired snake beam. The results of a simulation of this snake beam, using a Fresnel propagator, are shown in Fig. 2(c).

Although the overall beam is a collection of small sword beams, there is no restriction on the continuity or the order of these beams. Thus, one can create a morecomplicated three-dimensional patterns than just the simple snake beam. An example of such a structure, which belongs to the class of treelike focal lines, is shown in Fig. 3(b). This is again a three-swordbeam structure, but this time all three beams share a common end. The real part of the corresponding hologram's function is shown in Fig. 3(a).

The most direct way to implement these holograms is by gray-scale phase masks. ${ }^{5}$ As a result of mutual shifts among the various elements on the mask, there are intermediate opaque regions. It may be hard to form such nontransparent regions on the phase mask. However, our simulations indicate that even if these regions are completely transparent (and thus the mask is a pure phase-only mask) we still get the desired snake beam if it is located far away from the focus. That is because most of the light that comes from the uniform intermediate regions concentrates into the focus.

In conclusion, an arbitrary twisting paraxial focal line was introduced and demonstrated by two examples. This method is different by a few aspects from the technique proposed in Ref. 6. The method of Ref. 6 is based on one-dimensional Fresnel zone plates with a variable focal length. Therefore, unlike in our case, their focal line lacks a circular symmetry. It cannot be tilted by small angles from the optical axis, whereas our beam cannot follow large angles. Finally, in our system the superposed wave vector (of all the plane waves composing the snake beam) in every lateral plane is parallel to the beam direction. On the other hand, in Ref. 6 the overall wave vector is always perpendicular to the hologram plane, no matter what the focal line's direction.

We thank the reviewers for their valuable comments. This research was supported by the U.S. Army Research Office and the Advanced Research Projects Agency.

\section{References}

1. J. Rosen, B. Salik, and A. Yariv, J. Opt. Soc. Am. A 12, 2446 (1995).

2. J. Durnin, J. J. Miceli, Jr., and J. H. Eberly, Phys. Rev. Lett. 58, 1499 (1987).

3. J. Ojeda-Castañeda and L. R. Berriel Valdos, Opt. Lett. 13, 183 (1988).

4. J. Rosen and A. Yariv, Opt. Lett. 19, 843 (1994); R. Piestun and J. Shamir, Opt. Lett. 19, 771 (1994).

5. L. B. Lesem, P. M. Hirsch, and J. A. Jordan, Jr., IBM J. Res. Dev. 13, 150 (1969); T. J. Suleski and D. C. O'Shea, "Gray-scale masks for diffractive-optics fabrication. I. Commercial slide imagers," Appl. Opt. (to be published).

6. C. Frère and O. Bryngdahl, Opt. Commun. 60, 369 (1986). 